\title{
CDISC SDTM Device Properties Test Name Terminology
}

National Cancer Institute

\section{Source}

National Cancer Institute. CDISC SDTM Device Properties Test Name Terminology. NCI

Thesaurus. Code C111112.

Terminology associated with the device properties test name codelist of the Clinical Data Interchange Standards Consortium (CDISC) Study Data Tabulation Model (SDT M). 